\title{
Challenges of snow leopard conservation in China
}

\author{
Juan $\mathrm{Li}^{1,2,3^{*}}$, Lingyun Xiao ${ }^{1} \&$ Zhi Lu, \\ ${ }^{1}$ Center for Nature and Society, College of Life Sciences, Peking University, Beijing 100871, China; \\ ${ }^{2}$ Department of Environmental Science, Policy and Management, University of California, Berkeley, CA 94720-3114, USA; \\ ${ }^{3}$ Panthera, New York, NY 10018, USA; \\ ${ }^{4}$ Shanshui Conservation Center, Beijing 100871, China
}

Received Febuary 26, 2016; accepted April 20, 2016; published online April 29, 2016

\begin{abstract}
Citation: Li, J., Xiao, L., and Lu, Z (2016). Challenges of snow leopard conservation in China. Sci China Life Sci 59, 637-639. doi: 10.1007/s11427-016-5067-9
\end{abstract}

The snow leopard (Panthera uncial, Figure 1A) is a kind of beautiful big cat that is believed to originate on the Tibetan Plateau seven million years ago (Tseng et al., 2013), and now inhabits alpine and subalpine zones in the remote and rugged mountains on the Tibetan Plateau and its surrounding mountain ranges. Its range countries include Afghanistan, Bhutan, China, India, Kazakhstan, Kyrgyzstan, Mongolia, Nepal, Pakistan, Russia, Tajikistan, and Uzbekistan (Figure 1B) (Snow Leopard Network, 2014). Snow leopards mainly prey on mountain ungulates like blue sheep and ibex, but sometimes also prey on marmots and livestock. As an apex predator and keystone species, snow leopards play an important role in maintaining the stability of the unique mountain ecosystem they inhabit.

It is estimated that only about 4,000-7,000 individual snow leopards are living in the wild now (Snow Leopard Network, 2014). With the threats of habitat loss, depletion of wild prey and poaching, the snow leopard has been listed as Endangered by International Union for Conservation of Nature (IUCN) since 1972, and was included in Appendix I of Convention on International Trade in Endangered Species of Wild Fauna and Flora (CITES) in 1975 (Snow Leopard Network, 2014).

Snow leopards in China, which account for about $60 \%$ of the global snow leopard population, are mainly distributed in Qinghai Province, Tibet, and Xinjiang Autonomous Regions, and also discovered in Gansu, Sichuan and Yunnan

*Corresponding author (email: lijuan924@gmail.com)
Provinces, and Inner Mongolia Autonomous Region (Figure 1B). Snow leopards experienced a hard time from the $1950 \mathrm{~s}$ to the 1980s in China. They were hunted as a livestock predator and were an economically valuable species, so that they had been nearly eliminated in most of the range provinces, like Sichuan, Qinghai, Xinjiang and Inner Mongolia by the late 1980s ( $\mathrm{Li}$ and $\mathrm{Lu}, 2014)$. The fate of snow leopards in China was changed in the late 1980s, when they were listed as a Class I national protected animal in 1989. Several nature reserves were established step-by-step in the snow leopard range area, such as the Tomur National Nature Reserve in Xinjiang in 1985, Qilian Shan National Nature Reserve in Gansu in 1987, Qiangtang National Nature Reserve in Tibet in 1993, and Sanjiangyuan National Nature Reserve in Qinghai in 2000 (Figure 1B).

In the past two decades, China has made big improvements in the conservation of snow leopards, however, there are still several challenges. Poaching is still a great threat to snow leopards (Snow Leopard Network, 2014). Snow leopard skins were traditionally used by local people to make hats, coats or decorative wall mountings; snow leopard bones, together with other leopard bones, were mainly used as a substitute for tiger bones. Besides these traditional uses of snow leopard parts, which are strictly prohibited now, the snow leopard market may be shifting towards luxury products in recent years ( $\mathrm{Li}$ and $\mathrm{Lu}, 2014)$. Due to the high price of snow leopard parts and the huge profits, new cases of poaching and illegal trade occur every year. From 2013 to 2015 , at least twelve such cases were uncovered by the po- 

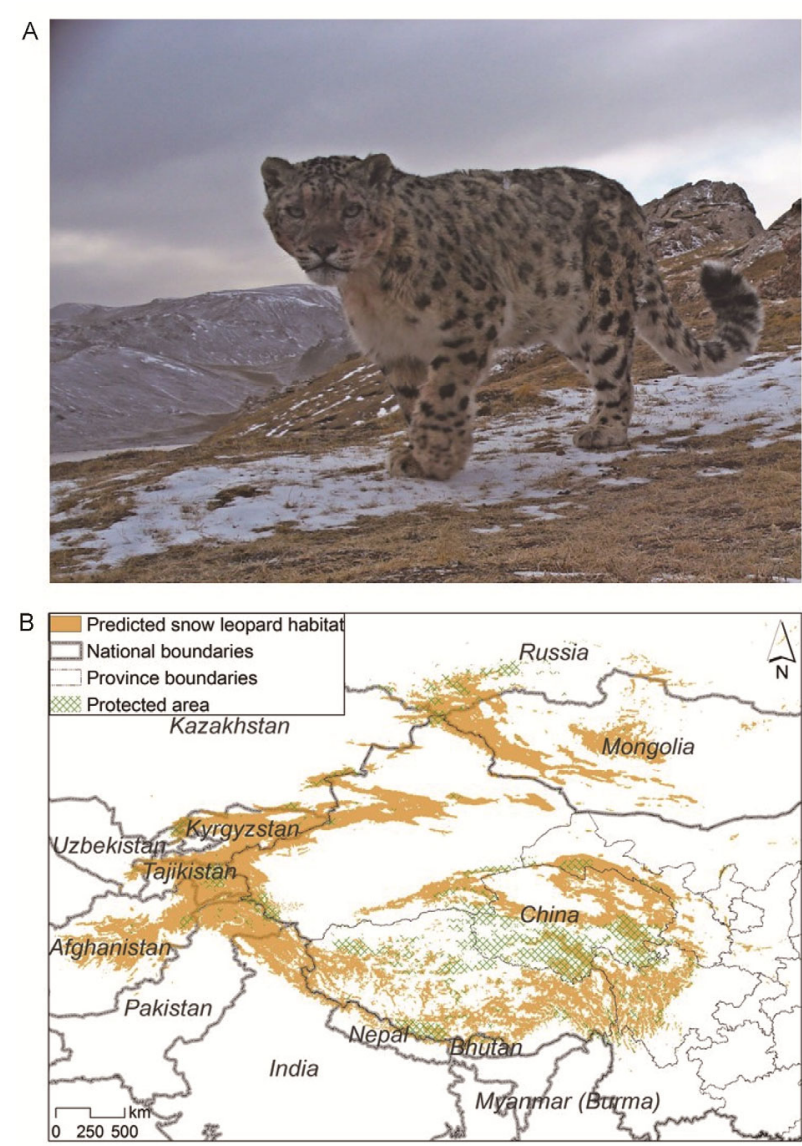

Figure 1 The snow leopard and its habitat. A, A snow leopard captured by our infrared camera traps in the Sanjiangyuan Nature Reserve, Qinghai Privince, China. B, Predicted global snow leopard habitat (Farrington and $\mathrm{Li}$, in press) and protected area

lice and reported by news. This involved three snow leopard cubs and at least thirteen pelts and seven carcasses of snow leopards. Considering that the reported cases represent only a part of the total cases, deaths of snow leopards from poaching pose one of the main threats to the already small population of snow leopards. Retaliatory killing, which is often intertwined with poaching, is also a potential threat, especially in areas that human-snow leopard conflicts are intense (Li et al., 2013).

Increasing human activities in snow leopard range areas is also a big challenge. Due to extreme harsh environment, people in the snow leopard range areas mainly live on animal husbandry and maintain a low population density. However, with the rapid economic development since 1980s, China launched mass infrastructure construction of railways and highways in almost all the snow leopard range area in China. This construction has led to habitat loss and fragmentation, squeezing the snow leopard's living space. Improved transportation has also led to a great increase of human activities in these areas, activities such as emergence of tourism and mining operations. These activities, especially the mining operations, are heavy burdens on the frag- ile ecosystem in the snow leopard range areas. Additionally, overgrazing by fast-growing livestock in the past decades caused grassland degradation in these areas, and this made the living condition of snow leopards and its prey even more difficult.

Along with economic development, modernization also challenges snow leopard conservation. The traditional way of life in the snow leopard range areas is mainly nomadism, with low consumption and low pollution. Now, more and more of the local people are settling down. Mobile vehicles and electronic products entered their life, making life in these areas more convenient, but also bringing pollution. The traditional culture and the Tibetan Buddhist beliefs, present in these areas, are eco-friendly and play an important role in snow leopard conservation (Li et al., 2014); yet, these are also impacted by modernization. All of these changes are potential threats to snow leopards.

Climate change is another challenge to snow leopard conservation. In the background of global warming, the warming rate of the mountain ranges where snow leopards inhabit has exceeded twice the Northern Hemisphere average in the past decades, and is predicted to continue or accelerate in this century (Liu and Chen, 2000). The alpine and subalpine zones where snow leopards inhabit are located between snow line and tree line. With the temperature rising, these zones will move up towards mountaintops, resulting in the loss of snow leopard habitat and habitat fragmentation. The eastern and southern edge of the Tibetan Plateau may be the most affected areas, including the Hengduan and east Himalaya mountain ranges in China (Farrington and Li, in press; Forrest et al., 2012). In addition, diseases, biotic interactions, and anthropogenic activities introduced by climate change might also bring other potential threats to snow leopards and associated species.

To cope with these challenges, the government needs to keep or strengthen law enforcement against poaching and snow leopard trades, and more importantly, should properly deal with the balance between economic development and ecological environment protection in snow leopard range areas. It is unreasonable, and also unrealistic, to request the local people to keep a hard but eco-friendly life. Sustainable conservation strategies should improve the life of both snow leopards and the local people. Comprehensive information on the status and ecology of snow leopards in China is required to develop such strategies. However, the snow leopard research in China started relatively late and the information is quite limited now. There was a little basic information from the national wildlife surveys in 1960s and George Schaller in 1980s (Schaller et al., 1988). Growing numbers of studies on snow leopards in China started in 2002, including population estimates, food habit, habitat selection and human-snow leopard conflicts. These studies were mainly conducted in Tomur Nature Reserve in Xinjiang (McCarthy et al., 2008), Sanjiangyuan Nature Reserve in Qinghai (Li et al., 2013) and Qilian Nature Reserve in 
Gansu (Alexander et al., 2015), which only covered a small part of the vast snow leopard range in China. Further studies should focus on the interactions between snow leopards and their sympatric animals, and furthermore, between snow leopards and the local ecosystem; impact of human activities and diseases on snow leopards also should be considered.

Besides the ecological information on snow leopards, understanding of the appeals and the culture of local people in the snow leopard range areas is also required. For example, in areas where snow leopards cause serious loss of livestock, compensation programs that aim to compensate the herders for their loss help alleviate human-snow leopard conflicts; in Sanjiangyuan area, with the help of Buddhist monks, the idea of snow leopard conservation has been accepted by most local people ( $\mathrm{Li}$ et al., 2014). It should be noted that in addition to the efforts of the government, non-government conservation organizations have also made great efforts and achieved great progress in snow leopard conservation. The advantage of the non-government conservation organizations is that they can provide more flexible and targeted conservation programs while considering the desires of local people.

Nowadays, snow leopards are attracting greater attention from the public, scientists, conservationists and the government both in China and worldwide. The Chinese government, along with other eleven snow leopard range countries made a Bishkek declaration to strive for snow leopard conservation in 2013. But snow leopard conservation still has a long way to go. Another big cat, the tiger, has ever owned its largest habitat and largest population in China, but extensive human activities almost extirpated its habitat and depleted its prey in China in the past century (Dinerstein et al., 2007). Taking a lesson from the fate of tigers in China would give snow leopards a more promising future.
Compliance and ethics The author(s) declare that they have no conflict of interest.

Alexander, J.S., Gopalaswamy, A.M., Shi, K., and Riordan, P. (2015). Face value: towards robust estimates of snow leopard densities. PloS One 10, e134815.

Dinerstein, E., Loucks, C., Wikramanayake, E., Ginsberg, J., Sanderson, E., Seidensticker, J., Forrest, J., Bryja, G., Heydlauff, A., and Klenzendorf, S. (2007). The fate of wild tigers. BioScience 57, 508-514.

Farrington, J., and Li, J. Climate change impacts on snow leopard range. In Snow Leopards, T.M. McCarthy, and D. Mallon, eds. (New York: Elesvier), in press.

Forrest, J.L., Wikramanayake, E., Shrestha, R., Areendran, G., Gyeltshen, K., Maheshwari, A., Mazumdar, S., Naidoo, R., Thapa, G.J., and Thapa, K. (2012). Conservation and climate change: Assessing the vulnerability of snow leopard habitat to treeline shift in the Himalaya. Biol Conserv 150, 129-135.

Li, J., and Lu, Z. (2014). Snow leopard poaching and trade in China 2000-2013. Biol Conserv 176, 207-211.

Li, J., Yin, H., Wang, D., Jiagong, Z., and Lu, Z. (2013). Human-snow leopard conflicts in the Sanjiangyuan Region of the Tibetan Plateau. Biol Conserv 166, 118-123.

Li, J., Wang, D., Yin, H., Zhaxi, D., Jiagong, Z., Schaller, G.B., Mishra, C., Mccarthy, T.M., Wang, H., Wu, L., Xiao, L., Basang, L., Zhang, Y., Zhou, Y., and Lu, Z. (2014). Role of Tibetan Buddhist monasteries in snow leopard conservation. Conserv Biol 28, 87-94.

Liu, X., and Chen, B. (2000). Climatic warming in the Tibetan Plateau during recent decades. Int J Climatol 20, 1729-1742.

Mccarthy, K.P., Fuller, T.K., Ming, M., Mccarthy, T.M., Waits, L., and Jumabaev, K. (2008). Assessing estimators of snow leopard abundance. J Wildl Manage 72, 1826-1833.

Schaller, G.B., Junrang, R., and Mingjiang, Q. (1988). Status of the snow leopard Panthera uncia in Qinghai and Gansu Provinces, China. Biol Conserv 45, 179-194.

Snow Leopard Network. (2014). Snow leopard survival strategy. Revised 2014 Version (Seattle: Snow Leopard Network).

Tseng, Z.J., Wang, X., Slater, G.J., Takeuchi, G.T., Li, Q., Liu, J., and Xie, G. (2013). Himalayan fossils of the oldest known pantherine establish ancient origin of big cats. Proc R Soc Lond 281, 20132686.

Open Access This article is distributed under the terms of the Creative Commons Attribution License which permits any use, distribution, and reproduction in any medium, provided the original author(s) and source are credited. 